\title{
A Method for Optimizing Opaque Filter Queries
}

\author{
Wenjia He, Michael R. Anderson, Maxwell Strome, Michael Cafarella \\ University of Michigan, Ann Arbor \\ \{wenjiah,mrander,mstrome,michjc\}@umich.edu
}

\begin{abstract}
An important class of database queries in machine learning and data science workloads is the opaque filter query: a query with a selection predicate that is implemented with a UDF, with semantics that are unknown to the query optimizer. Some typical examples would include a CNN-style trained image classifier, or a textual sentiment classifier. Because the optimizer does not know the predicate's semantics, it cannot employ standard optimizations, yielding long query times.

We propose voodoo indexing, a two-phase method for optimizing opaque filter queries. Before any query arrives, the method builds a hierarchical "query-independent" index of the database contents, which groups together similar objects. At query-time, the method builds a map of how much each group satisfies the predicate, while also exploiting the map to accelerate execution. Unlike past methods, voodoo indexing does not require insight into predicate semantics, works on any data type, and does not require in-query model training. We describe both standalone and SparkSQL-specific implementations, plus experiments on both image and text data, on more than 100 distinct opaque predicates. We show voodoo indexing can yield up to an $88 \%$ improvement over standard scan behavior, and a $79 \%$ improvement over the previous best method adapted from research literature.
\end{abstract}

\section{CCS CONCEPTS}

- Information systems $\rightarrow$ Query optimization; Multimedia and multimodal retrieval.

\section{KEYWORDS}

User-defined function (UDF) optimization; opaque filter query; hierarchical multi-armed bandit algorithm

Permission to make digital or hard copies of all or part of this work for personal or classroom use is granted without fee provided that copies are not made or distributed for profit or commercial advantage and that copies bear this notice and the full citation on the first page. Copyrights for components of this work owned by others than ACM must be honored. Abstracting with credit is permitted. To copy otherwise, or republish, to post on servers or to redistribute to lists, requires prior specific permission and/or a fee. Request permissions from permissions@acm.org.

SIGMOD '20, fune 14-19, 2020, Portland, OR, USA

(C) 2020 Association for Computing Machinery.

ACM ISBN 978-1-4503-6735-6/20/06 ..\$15.00

https://doi.org/10.1145/3318464.3389766
ACM Reference Format:

Wenjia He, Michael R. Anderson, Maxwell Strome, Michael Cafarella. 2020. A Method for Optimizing Opaque Filter Queries. In 2020 ACM SIGMOD International Conference on Management of Data (SIGMOD'20), Fune 14-19, 2020, Portland, OR, USA. ACM, New York, NY, USA, 16 pages. https://doi.org/10.1145/3318464.3389766

\section{INTRODUCTION}

Opaque filter queries constitute an important workload for processing unstructured text, images, and videos. In these queries, LIMIT clauses commonly occur as users navigate massive databases. For example, an engineer trying to improve a self-driving car might want to retrieve 100 images of crosswalks from a large database of dashcam video. She could train an image classifier that detects crosswalks, package it as a user-defined function (UDF), and then run the following SQL query:

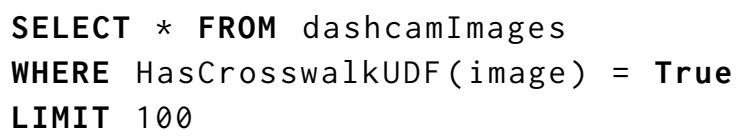

A naïve but traditional method [25] for these queries is to scan the whole database, apply the UDF to every row, and filter the result set according to the UDF's boolean answers. Obviously this method is time-consuming when the UDF runtime is long and the database is large. Canziani, et al showed that the deep model inference time per image is at least 5 milliseconds and can reach up to 200 milliseconds for models designed just five years ago [9]. Moreover, extremely large databases are easy to collect, not only from online text and image collections, but via the growing class of video applications; a single 30fps camera can obtain more than $2.5 \mathrm{M}$ frames a day.

Query optimization methods are crucial for good performance, but most have historically relied on semantic understanding of the query, which can pose a challenge when the query includes user-defined components. Recent research into optimizing UDF execution has tried to reduce query time through transforming or reordering UDFs for semantic equivalence $[35,36]$ and selecting indexes by code inspection [18]. However, these techniques remain limited, largely because of strong assumptions they make about the UDF's programming language, design, or possible semantics. These methods cannot help in the example below. 
Example 1. Harry is a self-driving car engineer whose job is to debug car behavior under specific failure scenarios. In this case, he needs to identify 100 examples of crosswalks in the video database, but in general there are always new scenarios to debug, so Harry cannot rely on a one-time video frame labeling step; instead, he must provide novel trained image classifiers as user-defined predicates and run opaque filter queries. Unfortunately, all real-life systems, whether relational or "big data" engines such as Hadoop [44] and Spark [48], execute these queries with a simple scan. Even worse, the inference time of each classifier invocation is long, making him wait a long time.

Technical Challenge - Existing methods cannot optimize our opaque filter query workload for two core reasons, both tied to the fact that the selection predicate is not only a UDF, but most likely a trained deep network. First, the selection predicates have semantics that are unclear to the database system and cannot be easily analyzed. As a result, it is not clear to the optimizer when a possible query optimization would be safe or effective. Second, the training procedure that yields the UDF selection predicate is likely an ongoing process, which constantly yields updated and novel predicates. As a result, it is not feasible to simply apply the predicate once to the entire database.

There has been some work on query systems for images and videos $[5,19,20]$ that do not have full semantic information about the data, but still save time as many traditional indexing methods do: by avoiding processing irrelevant data. Unfortunately, these methods are often not general-purpose, such as a frame-differencing method that works with video from stationary cameras [20]. Sometimes they can require expensive and error-prone online model retraining $[5,20]$.

Our challenge is to build an optimization method that (1) has no access to detailed UDF semantics, (2) is a generalpurpose approach that can work on many data types, and (3) avoids use of online model retraining.

Our Approach - In this paper, we propose a new mechanism for accelerating opaque filter queries that we call voodoo indexing. Like many optimization methods, it obtains speedups by avoiding processing irrelevant data that will not be returned to the user. Unlike past methods for optimizing UDF-based queries, voodoo indexing does not require any semantic insight into the UDF code. Further, voodoo indexing can work with any data type and does not require an online model retraining procedure.

Voodoo indexing's basic mechanism works in two phases. At index time, it clusters records into index groups so that similar objects are physically grouped together. At query time, it simultaneously builds a model of the rate at which different groups' records satisfy the UDF predicate, and also exploits the model to oversample records from the high-satisfy-rate groups. (Correspondingly, the method undersamples from groups where the UDF is satisfied comparatively rarely.) Voodoo indexing adapts this approach from the Zombie system, which was originally designed to support feature engineering tasks [4]; ours is the first general-purpose indexing mechanism with this two-phase architecture.

A core technical difficulty lies in quickly identifying and reading from the most-productive regions of the indexed database (that is, the database elements most likely to satisfy the UDF selection predicate). In addition to employing a multi-armed bandit policy [6] for deciding among index groups, voodoo indexing further adds a hierarchical sampling procedure. The core observation is that drawing a sample record from one index group yields evidence about the sampled group as well as weaker evidence about other similar groups. By exploiting this weak but plentiful evidence at query time, voodoo indexing can identify useful groups with very few samples, thereby yielding substantial performance gains beyond a naive adaptation of Zombie methods.

Example 2. In order to decrease waiting time, Harry employs voodoo indexing. At index time, voodoo indexing clusters together visually-similar frames from the dashcam video database. At query time, the system samples from a variety of index groups, sending each to the crosswalk detector UDF for evaluation. It finds that one index group contains a high fraction of data items that pass the selection filter, and oversamples from this group, quickly yielding 100 results.

Under this design, the system can identify many records that satisfy the UDF predicate early in the query execution process. It is intended to be most effective on "Goldilocks" LIMIT sizes that are neither extremely small nor extremely large. Extremely small result sizes will not give the system much opportunity to exploit the query-time information it collects, while extremely large result sizes will require the system to draw records from even very low-probability index groups. Fortunately, these mid-sized LIMIT sizes should be typical for the analytical and machine learning workloads we expect to see alongside UDF-based predicates.

Contributions - Our main contributions are as follows:

- We propose a novel method, voodoo indexing, for optimizing opaque filter queries. We model query processing as a hierarchical multi-armed bandit problem [47] and design an efficient algorithm for sampling.

- We evaluate our algorithm with real-world text and image databases, including MNIST, ImageNet and Yelp datasets, showing that voodoo indexing can yield up to an $88.2 \%$ improvement over standard scan, and a $79.0 \%$ improvement over adapted Zombie.

- We prototype our method in a standalone system as well one integrated with SparkSQL, and test its functionality on MNIST, showing up to $86.6 \%$ improvement. 


\section{PROBLEM FORMULATION}

In this section we introduce notation and a formal model for the opaque filter query optimization task, as well as for our proposed solution framework. All of the notation is listed in Table 1.

\begin{tabular}{|c|c|c|}
\hline Parameter & Description & Example \\
\hline$D$ (Given) & Raw dataset & Driving dashcam images \\
\hline$F$ (Given) & UDF predicate & Crosswalk detector \\
\hline$k$ (Given) & LIMIT number & 3 items \\
\hline$I$ (Designed) & Clusters & $\begin{array}{l}\text { Data clusters created by K- } \\
\text { Means or similar alg }\end{array}$ \\
\hline$T$ (Designed) & Dendrogram & $\begin{array}{l}\text { A balanced tree describing } \\
\text { image similarities }\end{array}$ \\
\hline$\mu$ (Designed) & Reward & $\begin{array}{l}\text { Crosswalk detector returns } \\
\text { True }\end{array}$ \\
\hline$n$ (Designed) & \# samples & 14 items are sent to $F$ \\
\hline
\end{tabular}

Table 1: Frequently used notation

\subsection{Problem Definition}

An opaque filter query is characterized by a 3-tuple of parameters $(D, F, k)$. The raw data $D$ is a large dataset that needs to be filtered, such as the dashcam image dataset in the above example. The function $F$ is user-defined function used as the selection predicate. It can be written using any language or programming framework. For example, it can be a TensorFlow or a PyTorch model, or even a hand-written piece of Python code. Items in dataset $D$ will be selected when function $F$ returns true. The LIMIT $k$ is the number of items that the query should return. Usually $k$ is driven by application concerns, such as the screen size, or the amount of time available to the user, or the required number of training examples for a downstream learning pipeline.

The value $n$ is the number of items in $D$ that are sent to $F$ at query time for evaluation, in hopes of finding $k$ items where $F$ returns true. We expect that $F$ will be time-consuming to run, so the runtime of the query is substantially driven by the time needed to run $n$ invocations of $F$. We can now define the optimization problem as follows:

Problem 1: Given input dataset $D$ and user-defined function $F$, find $k$ items that satisfy $F$ while minimizing $n$.

\subsection{UDF Optimization Strategy}

In order to solve the above problem, our model aims to intelligently choose the items in $D$ that are evaluated with $F$, such that the function returns false as infrequently as possible. At index time we organize the dataset $D$ into a similarity structure. For example, the structure can be a dendrogram $T$ in which the leaves are clusters of items, notated as $I$. At query time, our approach estimates the average utility of the clusters in order to select the useful ones to answer user's query. For each cluster it accumulates a reward $\mu$, computed as the fraction of records that return true when given to $F$. We can now describe our algorithmic task as follows: Design a similarity structure for raw dataset $D$, as well as a selection strategy, that will solve the above optimization task.

\subsection{Design Considerations}

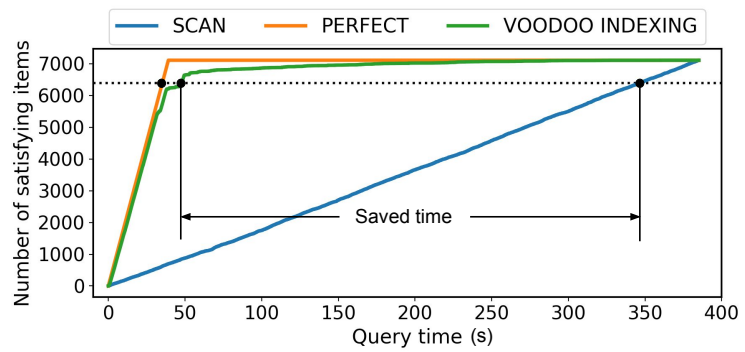

Figure 1: Typical execution curves for PERFECT, VOODOO and SCAN

Here we discuss some considerations in formulating a design.

LIMIT Size - Our goal is to optimize opaque filter queries with a LIMIT value $k$. Figure 1 shows how quickly different approaches can satisfy an opaque filter query on MNIST data [29]. The $\mathrm{x}$-axis describes query runtime, while the $\mathrm{y}$-axis indicates the number of data items retrieved by the system for which $F$ returns true. The dotted horizontal line indicates the query's LIMIT value (in this case, $90 \%$ of the total database size). This figure is derived from executing a real query. The PERFECT line indicates an ideal (but probably unrealizable) system that processes solely data items that return true when sent to the UDF predicate $F$. The VOODOO line indicates the method described in this paper.

Note that if the query's LIMIT value is extremely large or small, it is challenging for competing methods to improve over SCAN. Fortunately, we expect most $k$ values to fall in the broad middle due to databases' large size. Experiments in Section 6.2 show that while our method is effective across LIMIT values ranging from $10 \%$ to $90 \%$ of the satisfying item size, it shows best results for middle-range values.

UDF Behavior - We expect the UDF's runtime to be broadly consistent with published runtimes of trained classifiers running on GPU hardware. The longer the UDF runtime, the more useful it is to minimize invocations of $F$. We expect the UDF to be accurate enough to serve the purposes of the user's query; the work here does not try to improve the UDF's standalone accuracy.

\subsection{Illustrative Example}

In this section we illustrate the behavior of three possible implementations of opaque filter queries: standard SCAN, the Zombie method, and our proposed voodoo indexing method.

Example 1: SCAN. This example is shown in Figure 2 (a). Harry is now filtering crosswalk images by applying a learned 


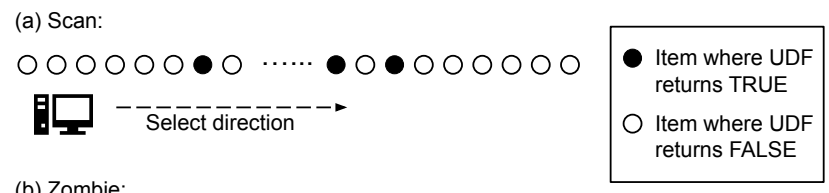

(b) Zombie:

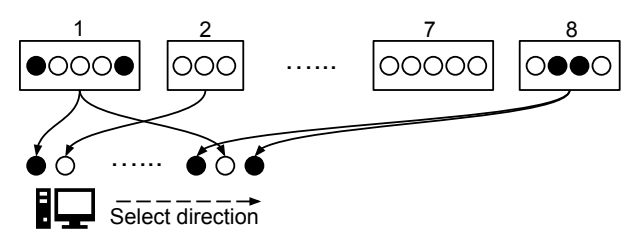

(c) Voodoo:

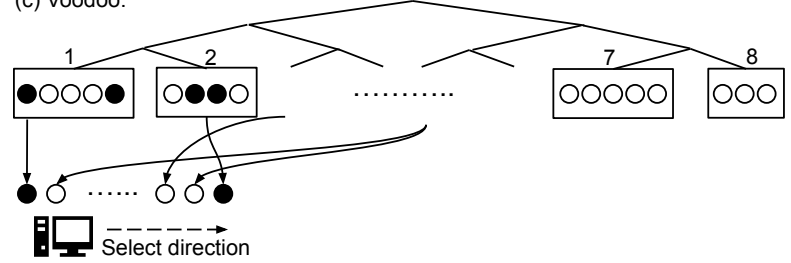

Figure 2: Three methods for the illustrative example

classifier as UDF, and has indicated a LIMIT value of $k=3$. There are 32 records in the database; 4 of them return True when sent to the UDF. In this case, the UDF needs to be applied 24 times in order to obtain 3 useful images.

Now consider the following example about the adapted Zombie method:

Example 2: ZOMBIE. This example is shown in Figure 2 (b). Harry now tries utilizing a research query system that employs a method adapted from the Zombie algorithm. At index time, these data records are clustered into 8 index groups. Some have a large fraction of data items where $F$ will return True. At query time, the system repeatedly draws each item from a chosen index group. By monitoring the rate at which index group yields a record where $F$ returns True, the UDF only needs to be applied 18 times in order to obtain 3 useful images (a 25\% reduction from standard practice).

Now consider our voodoo indexing approach:

Example 3: VOODOO. This example is shown in Figure 2 (c). At index time, the data records are again clustered into index groups. In addition, those clusters are placed in the leaves of a dendrogram, in which similar clusters are closer to each other in the tree. At query time, the system again repeatedly draws each item based on the dendrogram. In this way, the system can learn to ignore useless clusters (from cluster 3 through 8) with fewer accesses than in the above method. In this case, the UDF needs to be applied just 14 times in order to obtain 3 useful images (a $41.7 \%$ reduction from standard practice, and a $22.2 \%$ reduction from Zombie.)

These scenarios show actual examples on small datasets. Our method can show larger advantages when applied to larger databases, as shown in Section 6.

\section{BACKGROUND: MULTI-ARMED BANDITS}

Before presenting our work for optimizing opaque filter queries, we will introduce some needed background about the multi-armed bandit problem.

The multi-armed bandit problem is from the area of reinforcement learning [7]. An agent is confronted with a set of "one-armed bandits", or slot machines, each of which has a different probability of payout. The agent has a limited number of pulls to obtain payout from these machines, and could choose to spend those pulls identifying the best bandit (that is, exploring) or spend on the bandit currently known to be best (that is, exploiting). The goal of the problem is to balance these two motives to maximize the total payout.

The UCB algorithm [6], a formal way to simultaneously explore and exploit, is commonly used to solve this bandit problem. The upper confidence bound (UCB) of each slot machine's utility is composed of the current average reward (the exploitation term) and the one-sided confidence interval that may contain the true utility (the exploration term):

$$
U C B_{a, t}=\mu_{a}+\alpha \sqrt{\frac{2 \ln n}{n_{a}}}
$$

where $U C B_{a, t}$ is the upper confidence bound of machine $a$ at each time $t, \mu_{a}$ is the average reward of machine $a, \alpha$ is the exploration-exploitation balancing parameter, $n$ is the overall number of inserted coins, and $n_{a}$ is the number of coins inserted in machine $a$. According to the UCB algorithm, the machine that currently maximizes the upper confidence bound is always selected for the next action.

When we adapt this solution in our situation, each bandit corresponds to an index group, and each "pull" is the drawing of a data record from an index group. The reward at each time indicates whether the UDF predicate returns True for the chosen data record. We will discuss this bandit model in detail in the next section.

\section{ALGORITHMS}

Now we describe our novel voodoo indexing algorithm that helps users optimize opaque filter queries. In Section 4.1, we introduce a generalized version of Zombie that we call Zombie-G. Zombie was designed to solve a particular feature engineering task, which can be seen as an example of the generic opaque query optimization problem. In Section 4.2, we propose the basic version of voodoo indexing utilizing hierarchical multi-armed bandit algorithm. It samples from one cluster and sheds partial evidence about other clusters, leading to faster convergence on a good policy. In the remaining sections we discuss additional components that can benefit performance under particular situations. 


\subsection{Zombie-G}

Overview - Zombie-G contains two stages as shown in Figure 3: the indexing stage and the query processing stage. Its core idea is to construct a query-independent index before any query arrives, and then when executing queries select from high-payoff groups more frequently. Its structure is similar to two-phase operation in approximate query processing $[2,12]$. The algorithm is shown in Algorithm 1.

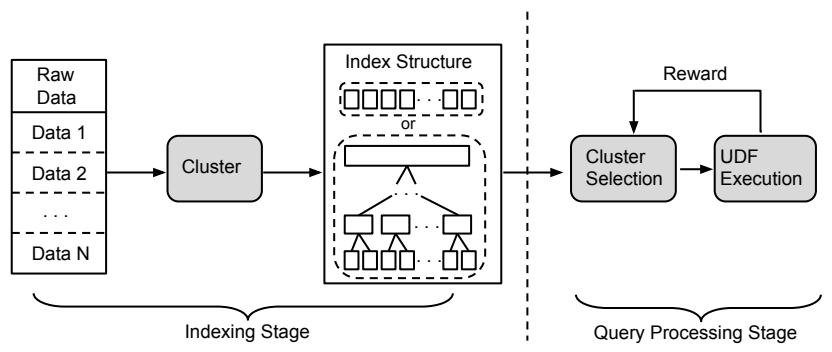

Figure 3: The architecture for opaque filter query optimization

Indexing - In the first stage, the raw dataset is re-arranged in a one layer index structure. A basic method is to cluster them into a set of index groups $I$ as shown in line 2 of $\mathrm{Al}-$ gorithm 1. This initial process tries to group similar data together and divide dissimilar data into different groups. For example, crosswalk images may be clustered together or texts about sports may be clustered together.

The clusters are not built with a particular query in mind: they simply group together data objects that are generally similar. We employ general-purpose clustering methods, such as K-Means. We also use task-independent features, such as pixel values or word frequency. Task-independent features are widely used; for example pixel value vectors as visual contents are correlated with digit classification [29], different object classifications [11,24], indoor-outdoor scene classification [42], et al. If insight about the database domain or query workload is known at indexing time, it can be used.

An important hypothesis of this approach is that downstream UDFs will filter records using properties that are captured in the index groups. This seems reasonable; most UDFs are not purely random and general features are related with a wide range of filter properties. If the UDF's classification and the index groups are correlated, then the query processing stage has a chance to identify these high-payoff groups. If the index happens to be totally unrelated with the UDF, Zombie-G will be unable to identify high-payoff groups, so its performance will be similar to (but not greatly worse than) simple scan.

We will examine only "insight-free" cases in Section 6, in which there is no knowledge ahead of time of the database domain or query workload.
Query Processing - In the second stage, the goal is to identify and draw from index groups that contain larger numbers of data records where $F$ returns True. We will treat a group's fraction of items where $F$ is True as its payoff. The true payoff of each group is an unknown parameter, because the UDF is potentially new with each query. We must simultaneously sample from groups to figure out their payoff, as well as use this information to improve future index group choices.

This problem is similar to multi-armed bandit problem, the purpose of which is to learn from "one-armed bandit" and maximize total rewards. We use the UCB algorithm to solve the problem. The algorithm works as follows: the UCB of every group is calculated in lines 4-6, the formula of which is the same as equation (1) in the last section, except that $a$ is the index for each group, $n$ and $n_{a}$ are the number of sampled records in total and for group $a$ respectively. $\mu_{a}$, the averaged reward of group $a$, is initialized to be 1 and updated as the system obtains counts during query execution. One item from the group with the highest UCB is selected in lines 7-8 (ties are broken by selecting randomly). This item is taken as the input of UDF $F$ to determine if it should be filtered, and to obtain the reward $\mu$ in lines 9-12. Related parameters are updated in line 13 for the next round. The algorithm terminates when the result set size meets the user's LIMIT requirement or when all the data have been selected.

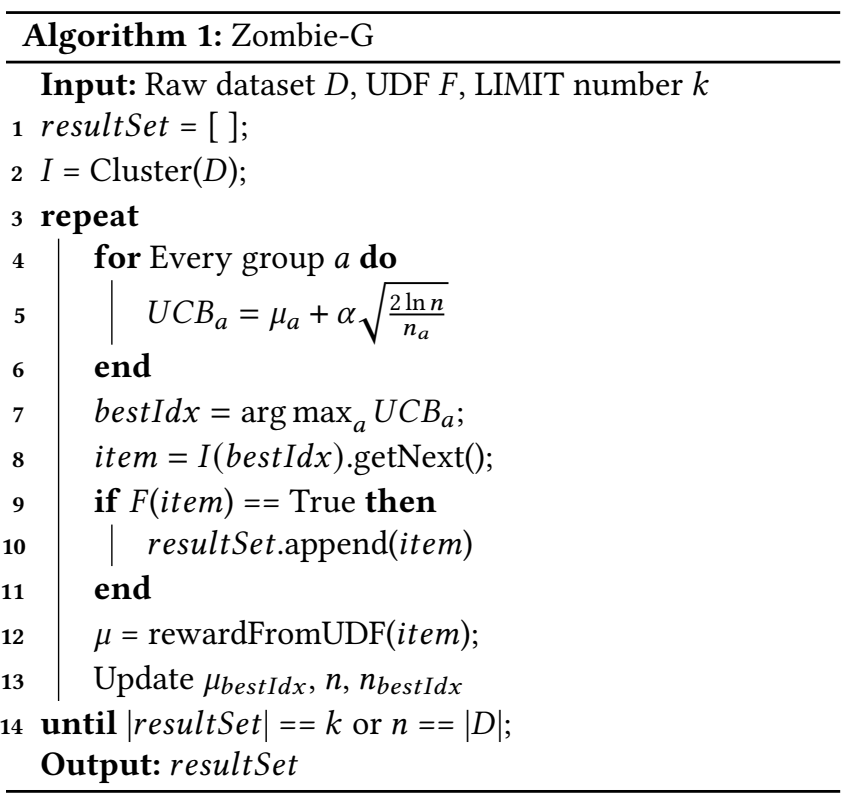

The Zombie-G algorithm can be useful (as we show in Section 2.4), but it has some weaknesses. In particular, it can take a long time to identify high-payoff index groups, especially when the space of bandits is large. Executing the user's query will entail broad sampling from many clusters 
for a long time, thereby processing many data items that do not satisfy the filter predicate. Only after sampling and processing records from many clusters does the system find high-value clusters to focus on. As we will see below, our voodoo indexing algorithm can do better.

\subsection{Voodoo Indexing}

The above algorithm treats each index group - each onearmed bandit - as entirely separate. But data items in different clusters still have a similarity relationship. Some clusters' contents are more similar to each other than to others, and a sample from one cluster may shed information about another. We can use this fact to better exploit the information we get from each sample - each "pull" of a bandit arm - and accelerate the agent's convergence to a good policy, thereby running the query much more quickly than Zombie-G can. Authors have previously proposed a hierarchical bandit algorithm [47], which mainly explores in a coarse low-dimensional space to greatly decrease the amount of unnecessary exploration and exploit fine-grained space to guarantee accuracy. This approach can potentially capture the correlations that exist between our data clusters. Different from the linear reward assumption and the feature vector representation in [47], we propose a new algorithm to leverages this coarseto-fine hierarchical idea.

Overview - Like Zombie-G, voodoo indexing has the twophase architecture shown in Figure 3. However, in order to leverage similarity between data clusters, we design a different index structure in the first stage and a different algorithm for item selection in the second stage. Algorithm 2 shows the voodoo indexing algorithm.

Indexing - In the indexing stage, the one-layer index structure in Zombie-G cannot capture the clusters' similarity. Instead, in voodoo indexing, raw data are arranged into a hierarchical structure $T$ in line 2 . To be specific, a dataset is first organized in clusters according to the Euclidean distance between items. These clusters are added as leaf nodes to a dendrogram, and then connected according to the Euclidean distance between groups' cluster centers. Each internal node can be thought of as a "virtual cluster" that contains all of the records in its child nodes. Various cluster method, such as K-Means, and dendrogram construction methods, such as agglomerative hierarchical clustering, can be utilized in this stage.

Query Processing - How can we use this structure to exploit the similarity between groups? Our novel algorithm is inspired by hierarchical optimistic optimization (HOO) in [8]. HOO is an arm selection policy designed for continuous reward functions, which we have modified for our discrete situation. First, the upper confidence bound is calculated for every group $a$ including leaf nodes and inner nodes at each time $t$ in line 4-6. In contrast to that previous work, inner nodes in the dendrogram only contain information of leaf nodes that have not yet been fully explored. Next, in line 7-10, the algorithm will choose a target cluster by starting at the root and repeatedly comparing the $U C B$ of the current node's nonempty children, selecting the winner:

$$
\text { BestChild }=\underset{c \in \text { nonempty children }}{\arg \max } U C B_{c}
$$

until reaching the bottom or a full index group that has not been touched before. Then after selecting the index group and updating the result set in line 11-14, the reward from applying the UDF to the selected item is received in line 15, which is given by:

$$
\mu_{\text {item }}= \begin{cases}1, & \text { if this item satisfies the predicate } \\ 0, & \text { otherwise }\end{cases}
$$

Subsequently, parameters $n, \mu_{a}$ and $n_{a}$ in the searching path are updated in line 16-19 in preparation for next $U C B$ update. Crucially, this procedure allows a sample from one leaf index group to influence future behavior for all subtrees containing it. The loop is repeated until meeting the stopping criteria.

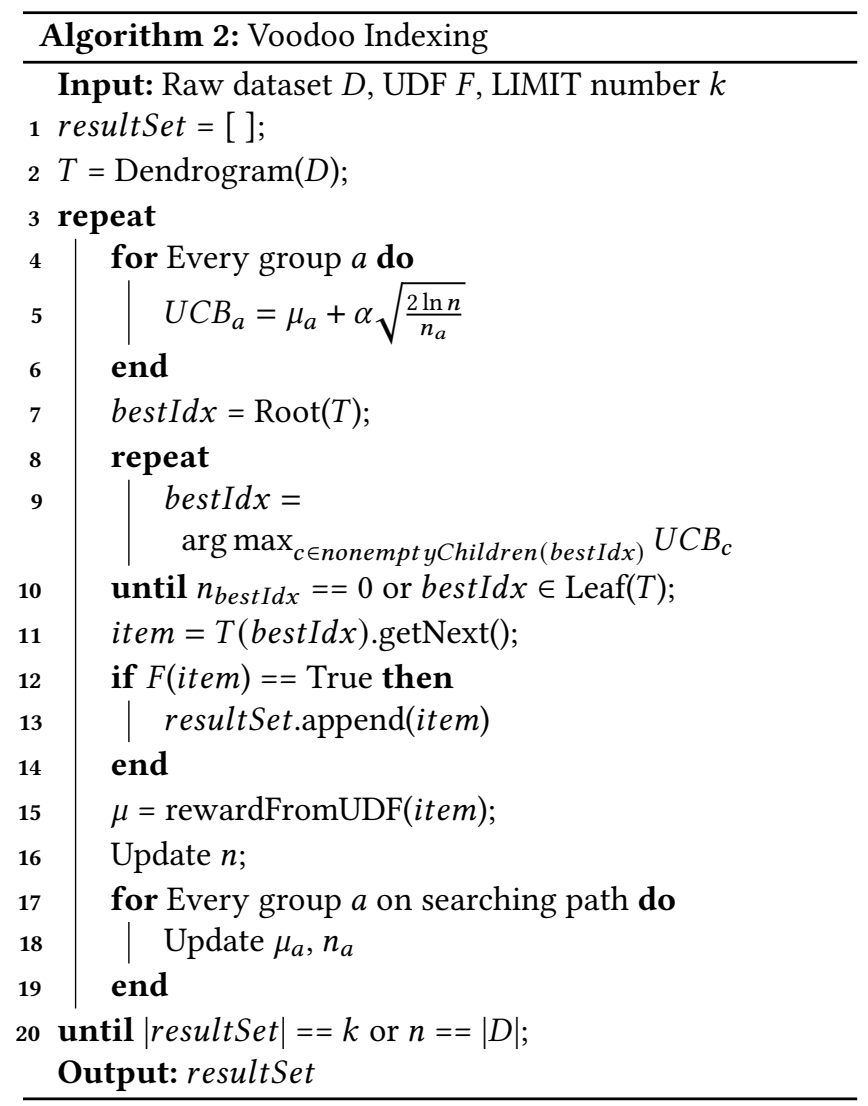




\subsection{Dynamic Index Recovery}

Voodoo indexing relies on the hierarchical index structure to better exploit sample information. However, this mechanism can only help if the dendrogram carries useful information. Dendrogram examples of different qualities are shown in Figure 4, where index groups that satisfy the UDF predicate $100 \%$ of the time are indicated in black, the $50 \%$ level is indicated in grey, and $0 \%$ is indicated in white. Even though every example tree is composed of the same clusters, not all of the trees are useful. In the good dendrogram, low-payoff clusters are concentrated in the right subtree, so only a few samples in the right subtree can tell the algorithm to decrease sampling frequency for these clusters. In the useless dendrogram, low-payoff clusters are intermixed with high-payoff clusters, so high-level internal nodes of the dendrogram provide almost no useful information. In the bad dendrogram, the low overall payoff of the right subtree will hide the actually best cluster, so the algorithm may actively avoid sampling from this high-payoff cluster.

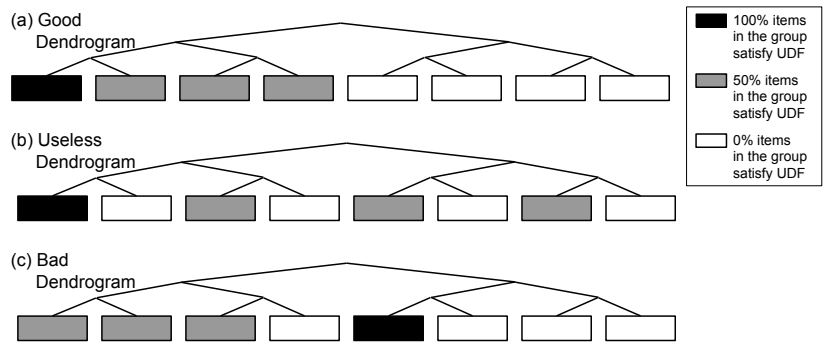

Figure 4: Dendrogram examples of different qualities

Without any evidence about the user's UDF filter, we can only use index-time information when constructing the dendrogram. Unfortunately, if this dendrogram does not group together clusters with similar filter behavior, a sample from one cluster does not give useful information about its sibling cluster, as with the last two dendrogram types above, and so voodoo indexing does not yield speedups. It can potentially do worse than Zombie-G or even a simple scan.

However, we note that as the user's query executes, we gain information about cluster similarity that was not available at index time. It may be possible to use this information to dynamically construct a new dendrogram that will help later stages of voodoo indexing execution. Algorithm 3 is our implementation of dynamic voodoo. It is the same as algorithm 2 in the indexing stage in line 2 . However, in the second stage, rather than retain the initial dendrogram for the entire execution, this algorithm may switch to a new one. If the algorithm detects that it is finding good data items less efficiently, possibly due to the inappropriate dendrogram structure, it holds a "contest" to determine whether it should switch to a new dendrogram. Two dendrograms compete in the contest: one is the initial dendrogram computed at index time, and the other is generated by sorting the leaf clusters by average observed rewards. A certain number of items are sampled from these two dendrograms separately by executing voodoo indexing, and the accumulated rewards are compared in lines 7-14. The algorithm will switch to the better dendrogram, until either a new contest is found to be needed, or the query terminates.

Although drawing items from the discarded dendrogram during the contest represents extra overhead, the predicate answers for these items can be stored for future sampling to decrease UDF running times. This is a greedy algorithm that makes the current optimal decision when unsatisfactory performance occurs. Even though it is possible that the greedy version may lead to a local optimum, comparing against the initial dendrogram guarantees that the dynamic version would not be worse than basic voodoo indexing.

The dynamic recovery algorithm requires setting a few parameters to determine the frequency of running each contest, and how long each contest should be run. In order to formulate a policy that permits the system to set these parameters automatically, we tested a wide range of parameter values on various workloads. The policy sets the balancing parameter $\alpha$ to a larger (1) value for smaller datasets (size $\leq 100,000)$, and smaller (0.1) for larger datasets (size > 100,000). It runs a contest no more frequently than after processing a $5 \%$ chunk of the database. It runs a contest if the last $5 \%$ of data yield UDF-satisfying data records at a rate of less than $80 \%$ of the previous $5 \%$ chunk. Running a contest entails processing $1 \%$ of the database through each candidate dendrogram.

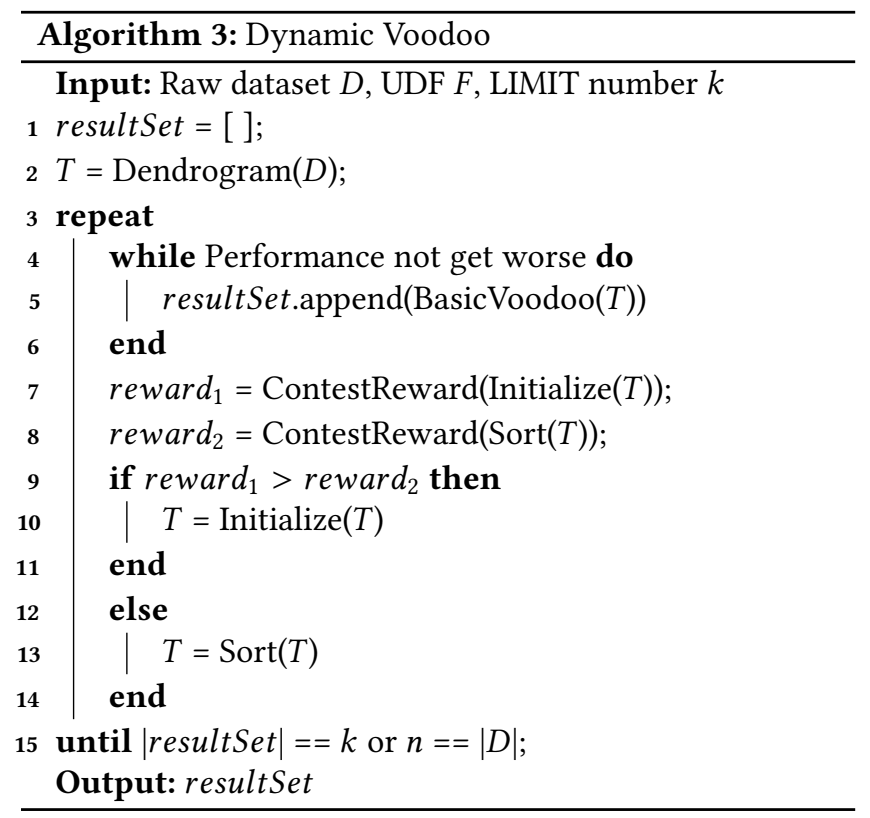




\subsection{Scan Failover}

A good dendrogram can help accelerate data selection. However, sometimes it may not be possible to identify an effective dendrogram. In some cases it may unfortunately be true that our method's basic hypothesis - that the UDF predicate yields results correlated with one or more the index groups is false. There is no correlation between what the user wants and our index structure. In these cases, the best thing we can do is to switch over to standard scan in order to avoid the additional overhead associated with our method. Therefore, we design a new component to detect when this failover is appropriate.

After running query processing for a certain period of time (we set it to be $10 \%$ of dataset size) our detector works to compare the current ratio of the number of items where $F$ returns True to number of processed items against that in simulated scan performance. The only challenge is how to determine a simulated scan sample size to minimize the sample overhead while accurately reflecting scan's expected performance. Confidence intervals can be leveraged to tackle this problem. We assume every item's predicate answer obeys the Bernoulli distribution $B(p)$ identically and independently, in which $p$ is the true ratio in the scan process that we want to estimate. Given the expectation $p$ and standard deviation $\sqrt{p(1-p)}$, we can get the convergence property according to the central limit theorem [27]:

$$
\frac{\sqrt{n}(\hat{p}-p)}{\sqrt{p(1-p)}} \stackrel{d}{\rightarrow} \mathcal{N}(0,1)
$$

where $\hat{p}$ is the sample average to estimate $p$. Therefore, the sample size under $95 \%$ confidence level should be:

$$
n=\frac{1.96^{2} p(1-p)}{(\hat{p}-p)^{2}}
$$

where 1.96 is the $z$-score. In our system, the margin $|\hat{p}-p|$ in the denominator is set to be half of $p$ for small $p(<10 \%)$ and one fifth of $p$ for large $p(>10 \%)$. We hypothesize that the true ratio $p$ equals the ratio $\bar{p}$ in voodoo indexing to determine the sample size $n$. After $n$ scan samplings, the confidence interval $\left[\hat{p}-1.96 \sqrt{\frac{\bar{p}(1-\bar{p})}{n}}, \hat{p}+1.96 \sqrt{\frac{\bar{p}(1-\bar{p})}{n}}\right]$ is supposed to contain the mean $\bar{p}$ under $95 \%$ confidence level. If $\bar{p}<\hat{p}$, the midpoint of the interval, it is highly probable that the true parameter $p \geq \bar{p}$, which means simple scan sampling is not worse than voodoo indexing, so we stop our voodoo indexing algorithm and switch to scan.

\subsection{Batch Mode}

In the previous algorithm, we selected only one item at each time, which fits the tuple-level iterator model that is common in database software. However, this method leaves unexploited advantages of batch-loading multiple items at once into GPU memory. Batch loading is a common low-level optimization for GPU workloads [45]. It has been widely used in neural network training in order to improve memory utilization and the parallel efficiency of matrix multiplication. We would like to leverage this optimization in our algorithm. We therefore slightly modify voodoo indexing during query processing to draw a batch of items from the index groups with the highest UCBs in each round, and feed into the UDF predicate a batch at a time.

The disadvantage of batch mode is that UCBs cannot be updated after each choice: the top picks in each round might be out of date. More concretely, in a particular batch of sampled items, the second pick cannot benefit from the learned information from processing the first pick. Nevertheless, batch mode can still help reduce runtime dramatically, which will be discussed in Section 6.

\section{SYSTEM PROTOTYPES}

We embodied our voodoo indexing algorithm in two prototype systems: a standalone Python implementation and an implementation integrated with SparkSQL. The former allows us to conduct detailed experiments on the algorithm's behavior, while the latter allows us to demonstrate voodoo indexing in a real-life working database system.

At a high level, our SparkSQL implementation allows users to ingest dataset files like they would for any standard SparkSQL database. Before any queries arrive, our system clusters the data elements into index groups. The indexed dataset now contains an additional field that identifies, for each row, an index group ID.

Standard SparkSQL behavior when processing an opaque filter query is to use an RDD iterator to scan the dataset in sequence, applying the UDF selection predicate to each element, and deciding whether to put the row into the result set. In contrast, we implemented a custom Scala SparkSQL operator that repeatedly interacts with a voodoo indexing backend, which tells the custom operator which group ID it should read next. This backend implements the index group choice procedure from Algorithm 2 in Section 4.2. Each step of this iterator also updates the voodoo indexing model about whether the last index group decision yielded a positive or negative result from the UDF predicate. (That is, it updates whether the bandit arm pull yielded a reward.)

We also implemented a new SparkSQL operator to perform correct but basic early stopping in LIMIT queries, regardless of whether the data is being processed in scan fashion or using voodoo indexing. (The standard plan generated by SparkSQL when performing LIMIT queries will not perform basic early stopping even when the LIMIT is satisfied, but rather will scan the entire dataset and then throw away results beyond the LIMIT.) 


\section{EXPERIMENTS}

We evaluated four core claims about voodoo indexing:

(1) Voodoo indexing performance is better than competing methods. Voodoo indexing can execute opaque filter queries more quickly than competing methods. This holds true across a range of real-world image and text datasets, as well as a range of user-defined selection predicates (that is, trained UDFs) (Section 6.2).

(2) All voodoo extensions yield benefits. The three extensions to voodoo indexing - dynamic index recovery, scan failover, and batch mode execution - yield better results than the naïve voodoo algorithm (Section 6.3).

(3) Voodoo is effective across many scenarios. Voodoo indexing can work over a very wide range of plausible real-world scenarios, which we demonstrate using a range of synthetic UDFs and clusterings (Section 6.4).

(4) Voodoo works in real systems, like SparkSQL. We show our approach yields direct runtime benefits when integrated with the real-life SparkSQL system (Section 6.5).

\subsection{Experimental Setting}

In this section, we describe our workloads, baseline methods, evaluation metrics, and experimental configuration.

Workloads - We evaluated our algorithm on multiple workloads. Each consists of a popular image or text dataset, plus a trained network to provide the UDF filter.

The MNIST database [29] contains 70,000 handwritten digit images. We trained a 10-digit image classifier, using the ResNet-18 [13] architecture modified to fit MNIST. It was trained from scratch, achieving $98.7 \%$ accuracy. It takes around $5 \mathrm{~ms}$ to process one image on a GPU. We used this classifier to build 10 different boolean UDFs: isDigi tZeroUDF(), isDigitOneUDF(), and so on. One such query takes the form:

\section{SELECT * FROM MNIST \\ WHERE isDigitZeroUDF(image) $=$ True}

When using ImageNet data[11], we use the Large Scale Visual Recognition Challenge 2012 (ILSVRC2012) [38] dataset, comprising 1,281,167 images in 1000 categories from ImageNet. It is widely used for image classification training. A quarter of the images were randomly chosen for our queries. We used the pre-trained ResNeXt-101 $(32 \times 8 \mathrm{~d})$ [46] model in PyTorch [34]. We also trained 80 binary classifiers for 20 randomly chosen categories (Table 2), varying the model architecture (ResNeXt-101 $(32 \times 8 \mathrm{~d})$ and VGG-19 [40]), learning rate (0.1 and 0.01 ) and optimizer (SGD and Adam). These models achieve $97.6 \%$ accuracy on average on the sampled ImageNet dataset. It takes the ResNeXt-based model about 36

\begin{tabular}{cccc}
\hline Category & Label ID & Category & Label ID \\
\hline American lobster & n01983481 & Lipstick & n03676483 \\
Dugong & n02074367 & Megalith & n03743016 \\
German shepherd & n02106662 & Pay phone & n03902125 \\
Greater Swiss & n02107574 & Pop bottle & n03983396 \\
mountain dog & & & \\
Great dane & n02109047 & Schooner & n04147183 \\
Fly & n02190166 & Screw & n04153751 \\
Bakery & n02776631 & Spotlight & n04286575 \\
Cuirass & n03146219 & Submarine & n04347754 \\
Fountain & n03388043 & Washer & n04554684 \\
Honeycomb & n03530642 & Corn & n12144580 \\
\hline
\end{tabular}

Table 2: The 20 randomly-selected ImageNet categories we used to train UDF models.

ms and takes the VGG-based model about $11 \mathrm{~ms}$ to process a single image on a GPU. Eight of the trained models never returned true for any tested input, so we removed them from the evaluation. We used these 72 trained classifiers and the 20 pretrained ones to build 92 boolean UDFs. One such query takes the form:

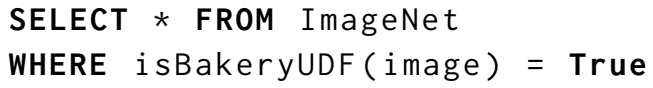

The Yelp dataset [1] contains textual reviews and numerical review scores, for local businesses. We selected 40,000 sentences, each of which contains at most 15 tokens, to be filtered for positive sentiment (having a review score $\geq 3$ ) or negative sentiment (having a review score $<3$ ). We used a pre-trained sentiment analysis model, based on a characterlevel LSTM neural network from the NLP library Flair [3]. The sentiment prediction model achieves $70 \%$ accuracy on the Yelp dataset and takes around $23 \mathrm{~ms}$ to process one item on a GPU. We used the model to build a single boolean UDF: isPositiveUDF(). The query is as follows:

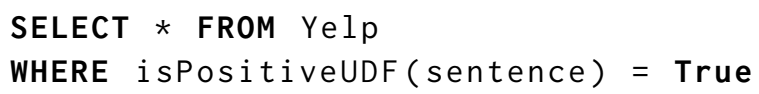

Baselines - We evaluated against two baselines.

- Scan - All DBMSes we are familiar with, including SparkSQL and PostgreSQL [31], will execute opaque filter queries with a simple scan, applying the UDF selection predicate to each row of data in sequence.

- Zombie-G - This is a generalized method from the Zombie system, introduced in Section 4.1.

Evaluation Metrics - We computed two core metrics to show the benefit of voodoo indexing: the total number of items that were processed by the UDF predicate, as well as total query time. The first metric reflects directly what voodoo indexing can change during execution. The second metric reflects our method's decisions, but also reflects the 


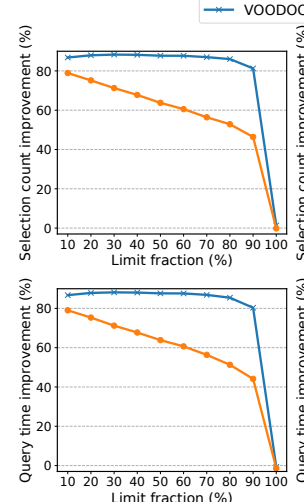

(a) MNIST
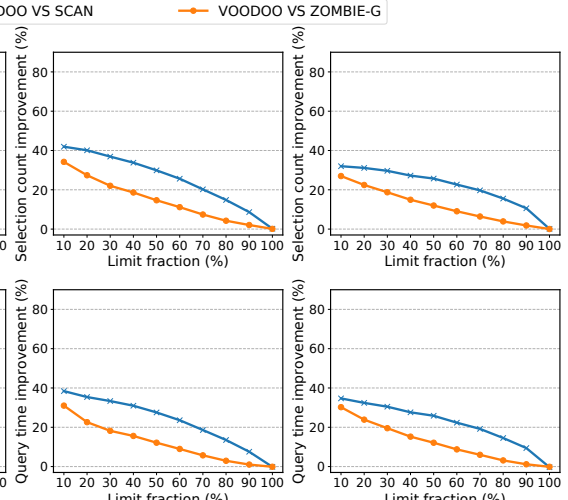

(b) ImageNet

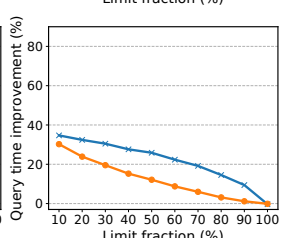

(c) Yelp
Lim fraction
Figure 5: Overall voodoo performance gains on MNIST, ImageNet and Yelp.

query's UDF runtime and our method's overhead. Although we report indexing time, we believe it is relatively unimportant compared to query time: the index structure is only computed once, then used for all queries that follow.

Experimental Configuration - We implemented our algorithm and all baseline methods in Python, and implemented SparkSQL-specific components in Scala. Experiments were run on a 64 -core $(2.10 \mathrm{GHz})$ Intel Xeon Gold 6130 server with 512 GB RAM and 4 GeForce GTX 1080 Ti GPUs.

\subsection{Overall Performance}

Summary - Our method voodoo indexing is better than competing efforts on a range of datasets and trained models. Over a range of query settings, voodoo indexing shows average query time improvements over competing methods of up to $88.2 \%$ on MNIST, $38.4 \%$ on ImageNet, and $34.7 \%$ on the Yelp dataset.

Overview - We evaluated voodoo indexing against Zombie$G$ and scan on our three off-the-shelf datasets. Before we executed any queries, the data was clustered as described in Section 4. We indexed each dataset just once; we used generic pixel value features when indexing MNIST and ImageNet, and tf-idf token features for Yelp. MNIST images are small (a $28^{*} 28$ grayscale image), so we used direct pixel values to produce a length 784 feature vector; we resized the higherresolution ImageNet images to grayscale $30 * 30$ images for a feature vector of length 900 . We clustered data using basic K-Means for MNIST and Yelp (with number of clusters set to 1000), and Mini Batch K-Means [39] for ImageNet (with number of clusters set to about 2500). We built initial dendrograms in all cases using agglomerative clustering.

We tested full dynamic voodoo indexing with scan failover. We did not use batch mode in these results, in order to make the various methods' behavior easier to understand. We cover batch mode performance in Section 6.3.3. We set all parameters according to the method in Sections 4.3 and 4.4.
We tested 103 distinct UDFs in total (10 MNIST UDFs, 92 ImageNet UDFs, and 1 Yelp UDF). These selection UDFs yield a wide range of observed selectivities: around 10\% (MNIST), from $0.1 \%$ to $8.0 \%$ (ImageNet), and around $50 \%$ (Yelp).

Results - We show improvement over competing methods in Figure 5. The figure shows the average improvement of voodoo indexing for all the UDFs in each dataset. The upper figures show reduction in processed item counts, while lower figures show reduction in query time.

Voodoo indexing shows improvements for all datasets and almost all LIMIT values, yielding up to an $88.2 \%$ improvement over scan and a 79.0\% improvement over Zombie-G. As we would expect, our method's advantage is smaller as the LIMIT size approaches $100 \%$; voodoo indexing needs to explore low-payoff clusters and may eventually process almost the entire dataset. In the case of MNIST, this led to slightly longer-than-competition query times when using a LIMIT of $100 \%$ of the database due to the extra overhead of UCB calculation and top-down group selection. However, even in this extreme case, voodoo indexing did not yield performance that was meaningfully below other methods.

For all datasets, our method's advantage over Zombie-G is lower than its advantage over scan, but is still substantial. Since both voodoo indexing and Zombie-G use the same sets of clusters and UDFs, these results show that our method's novel dendrogram approach is yielding real benefits in terms of reduced item selection and query runtimes.

Our method shows the best performance gains on MNIST. For a range of LIMITs from $10 \%$ to $90 \%$ of the data, voodoo indexing spends much less time than the other two methods. Because the MNIST UDF has a selectivity of $10 \%$, the best possible query processor - processing solely those data records where the UDF returns True - would yield a saving of $90 \%$. Our method saves $80.3 \%-88.2 \%$ of time compared to scan, close to that ideal. It saves $44.1 \%-79.0 \%$ of time compared to Zombie-G. Even when the user desires a small LIMIT size, for example 10\% (around 700 items), our method can locate and select good items very quickly (only around 920 selections and 5.0 seconds). In contrast, Zombie-G spends a long time early in execution trying to identify which index groups are valuable, showing most of its benefit only when the user needs larger amounts of data.

ImageNet data is much more complicated than the simple MNIST images, and has much finer-grained categories. But even in this challenging task, voodoo indexing clearly wins on average. It also beats scan in 83 out of the 92 individual UDFs. In nine cases the system detects that voodoo indexing is not successful, and so switches over to standard scan. Apart from extremely high-LIMIT cases, voodoo indexing always shows an improvement. It achieves a $18.6 \%-38.4 \%$ 


\begin{tabular}{cccc}
\hline \multirow{2}{*}{ Category @ LIMIT } & \multicolumn{3}{c}{ Query Time (s) } \\
\cline { 2 - 4 } & No-Switch & Switch & Relative Change \\
\hline Cuirass @ 30\% & 3219.9 & 2739.7 & $14.9 \%$ \\
Lipstick @ 30\% & 3965.0 & 3063.7 & $22.7 \%$ \\
Schooner @ 60\% & 3313.2 & 2598.2 & $21.6 \%$ \\
Screw @ 30\% & 3681.0 & 3216.0 & $12.6 \%$ \\
Spotlight @ 40\% & 4517.4 & 2946.1 & $34.8 \%$ \\
Corn @ 30\% & 4474.4 & 3849.2 & $14.0 \%$ \\
\hline
\end{tabular}

(a) The best LIMIT case

\begin{tabular}{cccc}
\hline \multirow{2}{*}{ Category @ LIMIT } & \multicolumn{3}{c}{ Query Time (s) } \\
\cline { 2 - 4 } & No-Switch & Switch & Relative Change \\
\hline Cuirass @ 70\% & 7203.9 & 7739.1 & $-7.4 \%$ \\
Lipstick @ 70\% & 7434.0 & 8267.7 & $-11.2 \%$ \\
Schooner @ 80\% & 5357.9 & 6146.2 & $-14.7 \%$ \\
Screw @ 90\% & 9537.7 & 9942.6 & $-4.2 \%$ \\
Spotlight @ 80\% & 6697.0 & 8001.6 & $-19.5 \%$ \\
Corn @ 90\% & 10491.0 & 10617.1 & $-1.2 \%$ \\
\hline
\end{tabular}

(b) The worst LIMIT case

Table 3: Compare voodoo indexing with and without the dynamic switch mechanism on ImageNet.

improvement over scan and 5.7\% - 31.0\% improvement over Zombie-G when LIMIT fraction is less than $80 \%$.

In addition to these image datasets, voodoo indexing can also work on the text dataset, Yelp, achieving a $19.2 \%-34.7 \%$ improvement over scan and $6.0 \%-30.2 \%$ improvement over Zombie-G when the LIMIT fraction is less than $80 \%$.

\subsection{Component Testing}

In this section we show that all three extensions to voodoo indexing are effective: dynamic index recovery, scan failover, and batch mode execution. In order to best illustrate their behavior when standard parts of voodoo indexing fail, we ran our experiments on the most challenging dataset: applying the pre-trained ResNeXt-101 model to filter each one of twenty categories from ImageNet dataset.

\subsubsection{Dynamic Index Recovery.}

Summary - Compared to naïve voodoo execution, using dynamic index recovery saves up to $34.8 \%$ in query time.

Overview - We tested how much dynamic index recovery can improve by trying to obtain better dendrograms midway through query execution. Whether using dynamic index recovery choice or not, we ran with the scan failover mechanism in place. We considered 200 scenarios: all 20 UDFs, at LIMIT levels ranging from $10 \%$ to $100 \%$. For each UDF, we identified the LIMIT setting that yielded the biggest benefit from dynamic index recovery, and the worst benefit from dynamic index recovery (which could be negative, i.e., the method worsens the performance).

Results - For six categories, using dynamic index recovery made a meaningful difference to query runtimes. (For the remaining 14 , using dynamic index recovery made no meaningful overall impact.) The best differences for these categories are shown in Table 3a. Compared to naïve voodoo indexing, dynamic voodoo indexing can save up to $34.8 \%$ query time. Note that this table shows the change derived from an individual dynamic index switch.

Although dynamic index recovery often helps overall, there may be certain settings where it actually yields worse results, as seen in Table 3b. (Remember that the greedy algorithm only examines query performance for a short period of time before deciding on a particular dendrogram for a long period.) But these bad scenarios are rare and small, and on balance dynamic index recovery is worthwhile. We believe this is because even if switching to a new index has an unfortunate impact, it can always switch back to a better dendrogram in the future.

6.3.2 Scan Failover.

Summary - The scan failover mechanism can successfully detect cases in the ImageNet data where voodoo indexing fails to yield a benefit, and then switch over to scan. This yields up to a $25.8 \%$ improvement in query time compared to dynamic voodoo indexing without the scan failover, and adds only $6.0 \%$ extra runtime on average compared to scan.

Overview - As described in Section 4.4, there may be situations in which the user's predicate is simply unrelated to the index groups. In this case, the best strategy is simply to fail over to scan. We ran dynamic voodoo indexing with and without the scan failover mechanism.

Results - Table 4 shows the results of these experiments for LIMIT setting that yields the best runtime improvement for scan failover. For five failed categories, we list the number of selected items used in the scan simulation process described in Section 4.4. We also show the runtimes for voodoo indexing, in no-failover and failover modes. As expected, this mechanism shows obvious speedup on all of these five failure cases, yielding up to $25.8 \%$ improvement. The overhead in implementing this method - the processing of the scan simulation items - entails selecting fewer than 20,000 items, or just $6 \%$ of the total dataset size. Therefore, after switching to scan, voodoo indexing's runtime of the cases in Table 4 is only $6.0 \%$ over scan's on average.

\subsubsection{Batch Mode.}

Summary - Batch mode can yield $61.7 \%$ - $66.2 \%$ query time improvements.

Overview - All of the previous experiments are run in single mode; that is, the method repeatedly (1) chooses a single data item to process, then (2) predicts which data item to choose next. However, for performance reasons we may want to load multiple data items into the GPU for batch processing, so as to fully utilize GPU RAM and data-parallel processing. 


\begin{tabular}{ccccc}
\hline \multirow{2}{*}{ Category @ LIMIT } & \multirow{2}{*}{ Simulation size } & \multicolumn{3}{c}{ Query Time (s) } \\
\cline { 3 - 5 } & & No-Failover & Failover & Relative change \\
\hline American lobster @ 50\% & 14898 & 6104.8 & 5835.3 & $4.4 \%$ \\
Dugong @ 40\% & 16390 & 5618.0 & 4624.5 & $17.7 \%$ \\
Greater Swiss Mountain dog @ 30\% & 15861 & 3849.0 & 2857.3 & $25.8 \%$ \\
Honeycomb @ 30\% & 18914 & 4585.6 & 3839.5 & $16.3 \%$ \\
Pop bottle @ 40\% & 19671 & 5596.6 & 4722.5 & $15.6 \%$ \\
\hline
\end{tabular}

Table 4: Comparing dynamic voodoo indexing with and without scan failover on five failure cases from ImageNet

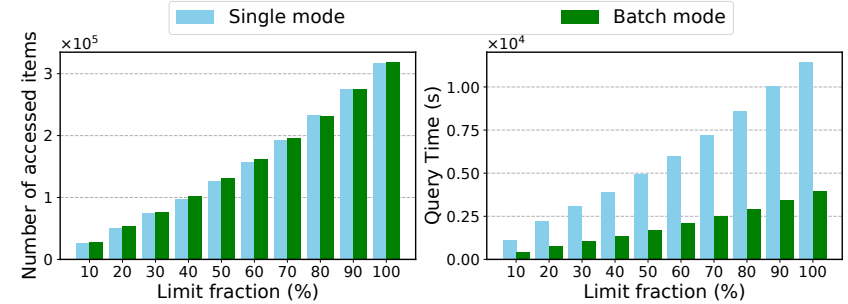

Figure 6: Batch vs single mode for voodoo indexing on the ImageNet dataset.

In this experiment, we modified the query time algorithm to choose 40 items at a time for processing. It loads all chosen items into GPU memory and applies the selection predicate to all 40 items. It then applies 40 updates to the bandit model.

Results - Figure 6 shows the averaged performance results, comparing dynamic voodoo indexing with scan failover in single mode and in batch mode. In the left figure, we can see that the two approaches process roughly the same number of items. It is not surprising that single mode in most cases processes fewer items than batch mode; the bandit model in single mode should be more accurate.

It is quite surprising that in some cases single mode may process more items than batch mode. We do not yet understand why this happens; our current hypothesis is that processing batches of items may have an impact similar to that of changing the $\alpha$ exploration/exploitation parameter, and that this has a positive impact in some situations.

But the right hand figure shows that the runtime advantages of batch mode are definitely worth any small losses encountered via choosing imperfect data items: batch mode can save $61.7 \%$ - $66.2 \%$ of query time compared to single mode for any LIMIT value.

\subsection{Varying Deployment Scenarios}

In this section, we generate synthetic UDFs, index structures, and datasets with different selectivities to show voodoo indexing's performance in the context of a wide range of deployment scenarios. We started with the same three realworld dataset. Unless otherwise stated in the text below, we filtered them for the same labels by using the same UDFs as in Section 6.2, and averaged the results. In these experiments we set the LIMIT value to $40 \%$ of the total number of satisfying data items, a moderate and representative LIMIT

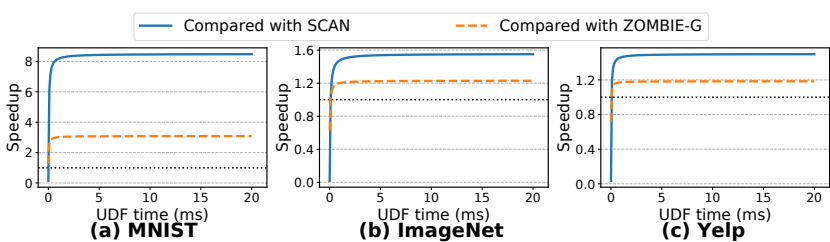

Figure 7: Speedup over Scan and Zombie-G for three datasets, varying the UDF time.

setting. We see that voodoo indexing is effective in most, but not all, plausible scenarios.

\subsubsection{UDF Execution Time.}

Summary - Voodoo indexing is effective for all UDF runtimes we would expect to see in common processing settings.

Overview - In this section, we tested the lowest bound of UDF times that still make voodoo indexing worthwhile compared with scan and Zombie-G. For very short runtimes, the overhead of voodoo indexing might not be worthwhile. We varied the UDF time from 0 to $20 \mathrm{~ms}$ for each dataset.

Results - Figure 7 shows the speedup factor of voodoo indexing over competing methods, with UDF runtime on the $\mathrm{x}$-axis. The black dotted line is the boundary when speedup is 1 . From this figure, we can see that our method's expected speedup is always greater than 1, except when the UDF runtime is very fast (under $1 \mathrm{~ms}$ ). At this stage, decreasing the number of predicate UDF invocations is not worth the extra overhead from the selection mechanism. As UDF runtime increases, voodoo indexing shows it is worth its extra overhead. Eventually, the method's speedup reaches a plateau where it is close to the observed ratio of UDF invocations.

\subsubsection{Index Structure Quality.}

Summary - Query performance varies with cluster quality; in some datasets, observed performance is close to what the best possible clustering might enable.

Overview - In this experiment, we varied the index group quality to see its impact on voodoo indexing and competing methods. We created synthetic indexes in the following manner: (1) we set the group size to be the same as the clustered structure, (2) created a random index structure by placing each data item into a single randomly-chosen cluster, (3) constructed a best index structure assigning data items to clusters according to their true labels. 


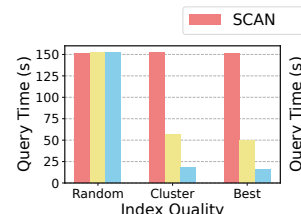

Index Quality
(a) MNIST

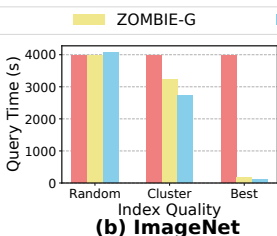

(b) ImageNet

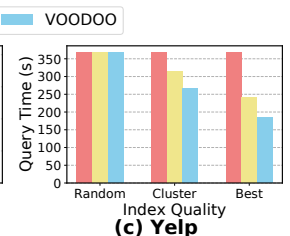

(c) Yelp
Figure 8: Analyze Voodoo's performance under different index structure quality on three datasets

Results - Perhaps unsurprisingly, Figure 8 shows that both voodoo indexing and Zombie- $\mathrm{G}$ are more effective when the index structure quality gets better. When the index structure is random, no method can do well, though at least the overhead of our method is minimal compared to scan. It is interesting to compare best performance to what can actually be obtained with the existing clustering. In Yelp and (especially) MNIST, the observed practical performance comes close to what is enabled by the best possible clustering. For ImageNet, the large gap between observed performance and the best suggests that our practical clusterings of ImageNet are worthwhile but far below what might be possible. This analysis gives reason to hope that with better cluster features, voodoo indexing might be able to obtain substantially better performance on ImageNet-linked queries.

\subsubsection{Selectivity.}

Summary - Voodoo indexing is effective under a very wide range of moderate UDF selectivities. It shows no worse performance than competing methods when UDF selectivities are extreme.

Overview - In this experiment, in order to test the range of UDF selectivities for which voodoo indexing is effective, we randomly selected ten binary ResNeXt-based UDFs in ten categories, from among the eighty trained models introduced in Section 6.1. We constructed new datasets from ImageNet to yield an effective selectivity of around $0.5 \%, 10 \%, 30 \%$ and $90 \%$ for these UDFs. We averaged the query time improvements over competing methods for each selectivity level.

Results - Figure 9 shows that for all tested selectivities, voodoo indexing is better than standard scan and Zombie-G. When the selectivity is in the middle range (e.g. 10\% and 30\% as shown in the figure), our method yields substantial benefits. These results are to be expected. When the selectivity is extremely large, scan is an extremely effective algorithm, and when selectivity is extremely low, it can be challenging to find satisfying data records even in a high-quality index.

\subsection{SparkSQL Experiments}

Summary - Our prototype system can work for real SQL query processing and can achieve up to an $86.6 \%$ improvement over SparkSQL.

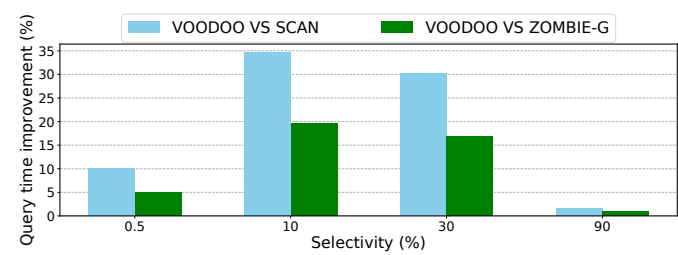

Figure 9: Query time improvement over competing methods on ImageNet, varying the selectivity.

\begin{tabular}{c|cc|cc|cc}
\hline \multirow{2}{*}{ LIMIT } & \multicolumn{2}{|c|}{ \# samples } & \multicolumn{2}{c|}{ T(s)(UDF=5ms) } & \multicolumn{2}{c}{ T(s)(UDF=10ms) } \\
\cline { 2 - 7 } & Voodoo & Spark & Voodoo & Spark & Voodoo & Spark \\
\hline $10 \%$ & $\mathbf{8 6 0}$ & 7248 & $\mathbf{7 . 4 0}$ & 36.43 & $\mathbf{1 1 . 7 0}$ & 72.67 \\
$20 \%$ & $\mathbf{1 5 8 5}$ & 14313 & $\mathbf{1 7 . 0 2}$ & 72.15 & $\mathbf{2 4 . 9 5}$ & 143.72 \\
$30 \%$ & $\mathbf{2 3 5 0}$ & 21371 & $\mathbf{2 2 . 9 2}$ & 107.53 & $\mathbf{3 4 . 6 7}$ & 214.39 \\
$40 \%$ & $\mathbf{3 0 5 1}$ & 28589 & $\mathbf{2 3 . 7 8}$ & 143.74 & $\mathbf{3 9 . 0 4}$ & 286.69 \\
$50 \%$ & $\mathbf{3 7 7 9}$ & 35479 & $\mathbf{3 1 . 1 6}$ & 178.17 & $\mathbf{5 0 . 0 6}$ & 355.57 \\
$60 \%$ & $\mathbf{4 4 9 7}$ & 42320 & $\mathbf{3 5 . 6 5}$ & 212.46 & $\mathbf{5 8 . 1 3}$ & 424.06 \\
$70 \%$ & $\mathbf{5 2 2 7}$ & 49306 & $\mathbf{4 0 . 1 2}$ & 247.41 & $\mathbf{6 6 . 2 5}$ & 493.94 \\
$80 \%$ & $\mathbf{6 3 0 7}$ & 56124 & $\mathbf{4 8 . 0 1}$ & 281.63 & $\mathbf{7 9 . 5 4}$ & 562.25 \\
$90 \%$ & $\mathbf{8 3 9 4}$ & 63154 & $\mathbf{8 2 . 6 9}$ & 316.71 & $\mathbf{1 2 4 . 6 6}$ & 632.48 \\
$100 \%$ & $\mathbf{6 7 0 4 8}$ & 69984 & 660.98 & $\mathbf{3 5 1 . 0 6}$ & 996.22 & $\mathbf{7 0 0 . 9 8}$ \\
\hline
\end{tabular}

Table 5: Results of our system and SparkSQL on MNIST

Overview - We tested our SparkSQL-specific system on 70000 images in MNIST dataset by running a SQL query with a synthetic UDF implemented as the predicate to filter digit 0 images. The executed query is:

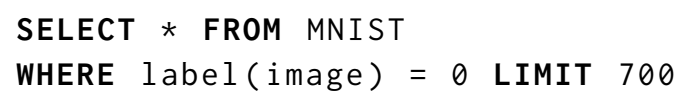

with varying LIMIT value. In order to show our system's performance under different levels of UDF complexity, we made the UDF label() returns ground truth and set the UDF time to be $5 \mathrm{~ms}$ and $10 \mathrm{~ms}$. Similar to previous experiments, the dataset is clustered into 1000 groups and arranged in a dendrogram, and the balancing parameter $\alpha$ is set to be 1 . We compared our system against a standard SparkSQL system that we extended with a LIMIT operator that stops scanning when the user query is satisfied.

Results - Table 5 compares our prototype system and SparkSQL in the terms of the number of items selected and the query time, with different kinds of UDFs. From this table, we can see that for different LIMIT levels, our system executes many fewer selections than standard SparkSQL, except the improvement when the LIMIT fraction is $100 \%$. In this case, voodoo indexing encounters the inevitable problem of lowpayoff cluster exploration. No matter if UDF execution time is $5 \mathrm{~ms}$ or $10 \mathrm{~ms}$, our system is much faster than SparkSQL when LIMIT is less than $100 \%$, yielding up to $86.6 \%$ improvement in query time. The saved UDF time can make up for the extra overhead in our system around item location and selection. And when UDF execution time is large, our system's strength is more obvious. 


\subsection{Discussion}

While our system can work for a wide range of opaque filter queries, it still has several limitations. Our mechanism may not be helpful when LIMIT values are extremely small, because of the time needed to identify high-value clusters. When the LIMIT value is extremely large, the system may also be slow, processing even low-payoff clusters. Fortunately, we believe that the broad range of middle-size LIMIT values will be popular and important, representing a good fit to analytical and ML style workloads.

The system may also be ineffective when the UDF returns TRUE extremely frequently or infrequently, because there is almost no difference between clusters' payoff, making cluster selection useless work and degrade to traditional scan. However, opaque filter queries with moderate selectivity are again likely to be a widespread workload: usually users would choose a related massive database consisted of items in more than one classes.

Overall, except for the above unusual situations, our mechanism is beneficial for common opaque filter queries on popular databases.

\section{RELATED WORK}

Several different research areas are related to our work, which we discuss in depth below:

UDF Optimization - Database researchers have been working on optimizing queries with UDFs for decades [14]. The main approaches can be divided into three categories. The first one is to analyze the semantic of UDF code so as to leverage traditional optimization techniques, such as sub-query optimization [35], operator reordering [15, 16, 36] and index selection $[10,18]$. However, due to the opaqueness of UDF code, some UDFs (e.g. with variant structures) cannot be automatically understood for optimization. The second one is to create new predicates for pre-filtering without careful semantic analysis [5, 19, 20,30]. Unfortunately, these works rely on historical queries, otherwise they need to train binary classifiers at cold start which stage can not be optimized. The third one is to sample data from indexed groups by learning mapping between UDF and groups at query time. Zombie [4] leverages this idea for feature engineering acceleration which can be modeled as a filter query with UDF.

Learned Indexes - To quickly locate data to improve the speed of query processing, database indexes are of vital importance. Voodoo indexing, learning the index information to instruct sampling, is similar to learned index methods. Database cracking [17] intends to learn from users' requests. As part of query processing, it cracks the database into pieces according to series of queries to maintain database indices. It assumes that the queries from users are unchanging or just changing a little over time. However, it may not be a proper assumption for UDFs. Learned index structures [22] attempts to enhance or replace traditional index structures (e.g. B-Tree-Index, Hash-Index and BitMap-Index) with machine learning models so as to decrease indexing time and lookup time significantly. However, this work can only be useful when queries can be understood by the optimizer.

Query Optimization with Machine Learning - With the development of reinforcement learning [41] and deep learning [28], these machine learning methods have been widely applied to query optimization. The learnable optimization methods include join ordering [23, 43], subquery representation [33], cardinality estimation [21], selectivity estimation [26] and index structures [22]. In addition to traditional queries, web query optimization for search engine is another promising use case [32,37]. A substantial improvement can be seen under the help of machine learning in these works. And our work is another kind of application designed for handling opaque filter queries.

\section{CONCLUSION AND FUTURE WORK}

As opaque filter queries in machine learning workloads become ubiquitous, an efficient filter strategy is required. However, due to its opaque semantic and unstable characteristic, real-world systems just execute these queries by simple scan. In summary, we present a novel two-phase indexing mechanism for opaque filter query optimization, which can select data that satisfies UDF predicate quickly, yielding to query time reduction. In addition, we build standalone and SparkSQL-integrated systems, and verify that both of them can achieve high performance on real-world datasets.

In this work, we focus on basic filter queries with one UDF predicate. We view this version of voodoo indexing mechanism as the initial step towards a family of methods for opaque query optimization. We believe it may be possible to apply our method to filter queries with multiple predicates by adjusting the reward function. Applying our method to aggregation queries that include a selection is likely to be straightforward, but using a UDF in the HAVING clause of an aggregation query is a serious challenge. This latter situation might appear in large machine learning workloads that use database queries to feed training data to a vast number of model production pipelines; we believe that examining the larger context in which opaque filter queries operate is a promising direction for future projects. Finally, we would like to address the optimization challenge for more elaborate queries such as JOIN queries. A possible way is to adapt our mechanism for cardinality estimation.

\section{ACKNOWLEDGMENTS}

This research is in part supported by NSF Award 1936940. 


\section{REFERENCES}

[1] 2015. Yelp dataset challenge. https://www.yelp.com/dataset/challenge.

[2] Sameer Agarwal, Barzan Mozafari, Aurojit Panda, Henry Milner, Samuel Madden, and Ion Stoica. 2013. BlinkDB: queries with bounded errors and bounded response times on very large data. In Proceedings of the 8th ACM European Conference on Computer Systems. ACM, 29-42.

[3] Alan Akbik, Duncan Blythe, and Roland Vollgraf. 2018. Contextual String Embeddings for Sequence Labeling. In COLING 2018, 27th International Conference on Computational Linguistics. 1638-1649.

[4] Michael R Anderson and Michael Cafarella. 2016. Input selection for fast feature engineering. In 2016 IEEE 32nd International Conference on Data Engineering (ICDE). IEEE, 577-588.

[5] Michael R Anderson, Michael Cafarella, German Ros, and Thomas F Wenisch. 2019. Physical representation-based predicate optimization for a visual analytics database. In 2019 IEEE 35th International Conference on Data Engineering (ICDE). IEEE, 1466-1477.

[6] Peter Auer, Nicolo Cesa-Bianchi, and Paul Fischer. 2002. Finite-time analysis of the multiarmed bandit problem. Machine learning 47, 2-3 (2002), 235-256.

[7] Sébastien Bubeck, Nicolo Cesa-Bianchi, et al. 2012. Regret analysis of stochastic and nonstochastic multi-armed bandit problems. Foundations and Trends ${ }^{\circledR}$ in Machine Learning 5, 1 (2012), 1-122.

[8] Sébastien Bubeck, Rémi Munos, Gilles Stoltz, and Csaba Szepesvári. 2011. X-armed bandits. fournal of Machine Learning Research 12, May (2011), 1655-1695.

[9] Alfredo Canziani, Adam Paszke, and Eugenio Culurciello. 2016. An analysis of deep neural network models for practical applications. arXiv preprint arXiv:1605.07678 (2016)

[10] Surajit Chaudhuri, Vivek Narasayya, and Sunita Sarawagi. 2002. Efficient evaluation of queries with mining predicates. In Proceedings 18th International Conference on Data Engineering. IEEE, 529-540.

[11] J. Deng, W. Dong, R. Socher, L.-J. Li, K. Li, and L. Fei-Fei. 2009. ImageNet: A Large-Scale Hierarchical Image Database. In CVPR09.

[12] Minos N Garofalakis and Phillip B Gibbons. 2001. Approximate Query Processing: Taming the TeraBytes.. In VLDB. 343-352.

[13] Kaiming He, Xiangyu Zhang, Shaoqing Ren, and Jian Sun. 2016. Deep residual learning for image recognition. In Proceedings of the IEEE conference on computer vision and pattern recognition. 770-778.

[14] Joseph M Hellerstein and Michael Stonebraker. 1993. Predicate migration: Optimizing queries with expensive predicates. Vol. 22. ACM.

[15] Fabian Hueske, Mathias Peters, Aljoscha Krettek, Matthias Ringwald, Kostas Tzoumas, Volker Markl, and Johann-Christoph Freytag. 2013. Peeking into the optimization of data flow programs with mapreducestyle udfs. In 2013 IEEE 29th International Conference on Data Engineering (ICDE). IEEE, 1292-1295.

[16] Fabian Hueske, Mathias Peters, Matthias J Sax, Astrid Rheinländer, Rico Bergmann, Aljoscha Krettek, and Kostas Tzoumas. 2012. Opening the black boxes in data flow optimization. Proceedings of the VLDB Endowment 5, 11 (2012), 1256-1267.

[17] Stratos Idreos, Martin L Kersten, Stefan Manegold, et al. 2007. Database Cracking.. In CIDR, Vol. 7. 68-78.

[18] Eaman Jahani, Michael J Cafarella, and Christopher Ré. 2011. Automatic optimization for MapReduce programs. Proceedings of the VLDB Endowment 4, 6 (2011), 385-396.

[19] Daniel Kang, Peter Bailis, and Matei Zaharia. 2018. Blazeit: Fast exploratory video queries using neural networks. arXiv preprint arXiv:1805.01046 (2018).

[20] Daniel Kang, John Emmons, Firas Abuzaid, Peter Bailis, and Matei Zaharia. 2017. Noscope: optimizing neural network queries over video at scale. Proceedings of the VLDB Endowment 10, 11 (2017), 1586-1597.
[21] Andreas Kipf, Thomas Kipf, Bernhard Radke, Viktor Leis, Peter Boncz, and Alfons Kemper. 2018. Learned cardinalities: Estimating correlated joins with deep learning. arXiv preprint arXiv:1809.00677 (2018).

[22] Tim Kraska, Alex Beutel, Ed H Chi, Jeffrey Dean, and Neoklis Polyzotis. 2018. The case for learned index structures. In Proceedings of the 2018 International Conference on Management of Data. ACM, 489-504.

[23] Sanjay Krishnan, Zongheng Yang, Ken Goldberg, Joseph Hellerstein, and Ion Stoica. 2018. Learning to optimize join queries with deep reinforcement learning. arXiv preprint arXiv:1808.03196 (2018).

[24] Alex Krizhevsky, Geoffrey Hinton, et al. 2009. Learning multiple layers of features from tiny images. (2009).

[25] Marco La Cascia and Edoardo Ardizzone. 1996. Jacob: Just a contentbased query system for video databases. In 1996 IEEE International Conference on Acoustics, Speech, and Signal Processing Conference Proceedings, Vol. 2. IEEE, 1216-1219.

[26] Seetha Lakshmi and Shaoyu Zhou. 1998. Selectivity estimation in extensible databases-a neural network approach. In VLDB. 623-627.

[27] Lucien Le Cam. 1986. The central limit theorem around 1935. Statistical science (1986), 78-91.

[28] Yann LeCun, Yoshua Bengio, and Geoffrey Hinton. 2015. Deep learning. nature 521, 7553 (2015), 436.

[29] Yann LeCun, Léon Bottou, Yoshua Bengio, Patrick Haffner, et al. 1998. Gradient-based learning applied to document recognition. Proc. IEEE 86, 11 (1998), 2278-2324

[30] Yao Lu, Aakanksha Chowdhery, Srikanth Kandula, and Surajit Chaudhuri. 2018. Accelerating machine learning inference with probabilistic predicates. In Proceedings of the 2018 International Conference on Management of Data. ACM, 1493-1508.

[31] Bruce Momjian. 2001. PostgreSQL: introduction and concepts. Vol. 192. Addison-Wesley New York.

[32] Rodrigo Nogueira and Kyunghyun Cho. 2017. Task-oriented query reformulation with reinforcement learning. arXiv preprint arXiv:1704.04572 (2017).

[33] Jennifer Ortiz, Magdalena Balazinska, Johannes Gehrke, and S Sathiya Keerthi. 2018. Learning state representations for query optimization with deep reinforcement learning. arXiv preprint arXiv:1803.08604 (2018).

[34] Adam Paszke, Sam Gross, Soumith Chintala, Gregory Chanan, Edward Yang, Zachary DeVito, Zeming Lin, Alban Desmaison, Luca Antiga, and Adam Lerer. 2017. Automatic differentiation in pytorch. (2017).

[35] Karthik Ramachandra, Kwanghyun Park, K Venkatesh Emani, Alan Halverson, César Galindo-Legaria, and Conor Cunningham. 2017. Froid: Optimization of imperative programs in a relational database. Proceedings of the VLDB Endowment 11, 4 (2017), 432-444.

[36] Astrid Rheinländer, Arvid Heise, Fabian Hueske, Ulf Leser, and Felix Naumann. 2015. SOFA: An extensible logical optimizer for UDF-heavy data flows. Information Systems 52 (2015), 96-125.

[37] Corby Rosset, Damien Jose, Gargi Ghosh, Bhaskar Mitra, and Saurabh Tiwary. 2018. Optimizing query evaluations using reinforcement learning for web search. In The 41st International ACM SIGIR Conference on Research \& Development in Information Retrieval. ACM, 1193-1196.

[38] Olga Russakovsky, Jia Deng, Hao Su, Jonathan Krause, Sanjeev Satheesh, Sean Ma, Zhiheng Huang, Andrej Karpathy, Aditya Khosla, Michael Bernstein, et al. 2015. Imagenet large scale visual recognition challenge. International journal of computer vision 115, 3 (2015), 211-252.

[39] David Sculley. 2010. Web-scale k-means clustering. In Proceedings of the 19th international conference on World wide web. ACM, 1177-1178.

[40] Karen Simonyan and Andrew Zisserman. 2014. Very deep convolutional networks for large-scale image recognition. arXiv preprint arXiv:1409.1556 (2014) 
[41] Richard S Sutton and Andrew G Barto. 2018. Reinforcement learning: An introduction. MIT press.

[42] Martin Szummer and Rosalind W Picard. 1998. Indoor-outdoor image classification. In Proceedings 1998 IEEE International Workshop on Content-Based Access of Image and Video Database. IEEE, 42-51.

[43] Immanuel Trummer, Samuel Moseley, Deepak Maram, Saehan Jo, and Joseph Antonakakis. 2018. SkinnerDB: regret-bounded query evaluation via reinforcement learning. Proceedings of the VLDB Endowment 11, 12 (2018), 2074-2077.

[44] Tom White. 2012. Hadoop: The definitive guide. " O’Reilly Media, Inc.".

[45] Matthias Wloka. 2003. Batch, batch, batch: What does it really mean In Presentation at game developers conference.
[46] Saining Xie, Ross Girshick, Piotr Dollár, Zhuowen Tu, and Kaiming He. 2017. Aggregated residual transformations for deep neural networks. In Proceedings of the IEEE conference on computer vision and pattern recognition. 1492-1500.

[47] Yisong Yue, Sue Ann Hong, and Carlos Guestrin. 2012. Hierarchical exploration for accelerating contextual bandits. arXiv preprint arXiv:1206.6454 (2012).

[48] Matei Zaharia, Mosharaf Chowdhury, Michael J Franklin, Scott Shenker, and Ion Stoica. 2010. Spark: Cluster computing with working sets. HotCloud 10, 10-10 (2010), 95. 